\title{
Difficulties in using the exact closed-form PEP formula for BER calculation of S-T codes
}

\author{
X. G. Dai, Z. Zhang, S. W. Cheung and T. I. Yuk
}

\begin{abstract}
The formula for the exact union bound (EUB) on BER based on the exact closed-form Pair-wise Error Probability (PEP) is most accurate for calculating the bit-error-rates (BERs) of Space-Time (S-T) codes in Multiple-Input Multiple Output (MIMO) systems. In this paper, it is shown that using the exact closed-form PEP formula for numerical calculations of the PEP or the EUB on BER could produce negative results. The formula for the exact closed-form PEP is therefore re-examined and explanations for the problem are given. A simple trick is suggested to reduce the effect of the problem. A new upper bound on PEP derived using the HÖlder Inequality is proposed to replace the exact closed-form PEP formula. Numerical calculation results show that the union bound based on the proposed new upper bound on PEP is a very tight bound to the EUB and doesn't have the problem of negative results.
\end{abstract}

\section{Index Terms - exact PEP, union bounds on BER, MIMO}

\section{INTRODUCTION}

The Pair-wise Error Probability (PEP) has been considered as an essential tool for bit-error rate (BER) calculation and construction of Space-Time (S-T) codes [1] for use in MultipleInput Multiple-Output (MIMO) systems. Union bounds (UB) based on different upper bounds of PEP were proposed and studied in [1-4]. The exact closed-form formula of PEP was developed in $[5,6]$. Theoretically, the UB based on the exact PEP, here called the exact union bound (EUB), is the tightest upper bound and will produce the most accurate results for biterror rates. However, as we show in this paper, there are difficulties in using the exact PEP formula for numerical calculations of bit-error rates (BERs) because the exact PEP formula derived in $[5,6]$ is very sensitive to the computational precisions of the computer programs used. For computer programs with limited precisions, the PEPs calculated are inaccurate and sometimes have negative values. This paper proposes a new upper bound on PEP derived using the HÖlder inequality and the corresponding new union bound (NUB) for calculations of BERs. Results of numerical calculations show that this NUB is a very tight upper bound to the EUB and can be used to calculate the BER accurately without having the same computational precision problem of the EUB.

The rest of this paper is organized as follows. The system model used for studies is defined in Section II. The exact PEP and the corresponding UB on BER are briefly described in Section III. In Section IV, we explain the difficulties in using the exact PEP formula for numerical calculations of BERs. A new upper bound on PEP is proposed and comparison with EUB is made in Section V. Section VI concludes the paper.

\section{SYSTEM MODEL}

The system model used for the study is an $N_{t} \times N_{r}$ MIMO system with $N_{t}$ transmit antennas and $N_{r}$ receive antennas, over a quasi-static Rayleigh fading channel. The $N_{r} \times T$ received signal matrix $\mathbf{R}$ is given by:

$$
\mathbf{R}=\mathbf{H C}+\mathbf{W}
$$

where the entries of $\mathbf{H} \in \mathbb{C}^{N_{r} \times N_{t}}$ represent the channel coefficients which are assumed to be perfectly known at the receiver but not at the transmitter. The elements in $\mathbf{H}$ are assumed to be static within a transmission block and are independent identical distributed (i.i.d.) complex Gaussian variables with zero mean and variance 0.5 for the real and imaginary parts. $\mathbf{C} \in \mathbb{C}^{N_{t} \times T}$ is the codeword matrix with block length $T$. Each of the elements $\left\{c_{n, t}\right\}$ in $\mathbf{C}$ is a coded symbol transmitted from the $n^{\text {th }}$ transmit antenna in the $t^{\text {th }}$ bit-time interval and having average symbol energy $E_{b} . \mathbf{W} \in \mathbb{C}^{N_{r} \times T}$ represents the complex additive white Gaussian noise (AWGN) matrix with elements being i.i.d. and following the normal distribution $N_{\mathrm{C}}\left(0, N_{0}\right)$.

\section{EXACT PEP AND UNION BOUND}

The PEP is denoted as $P_{e}(\mathbf{C} \rightarrow \hat{\mathbf{C}})$, where $\hat{\mathbf{C}}$ is any codeword in the codebook other than $\mathbf{C}$. In $[5,6]$, the exact $\mathrm{PEP}$ was derived in an integral form as

$$
P_{e}(\mathbf{C} \rightarrow \hat{\mathbf{C}})=\pi^{-1} \int_{0}^{\pi / 2}\left\{\prod_{r=1}^{K}\left(1+\left(4 \sin ^{2} \theta\right)^{-1} \lambda_{r} S N R\right)^{-N_{r}}\right\} d \theta
$$

where $\lambda_{1} \ldots \lambda_{K}$ are the $K$ non-equal positive eigenvalues of the codeword difference matrix $(\mathbf{C}-\hat{\mathbf{C}})(\mathbf{C}-\hat{\mathbf{C}})^{*}$, with $*$ denoting the transpose conjugate and $S N R=E_{b} / N_{0}$.

Two possible ways have been proposed to evaluate the exact PEP in (2), depending on whether the eigenvalues of the codeword difference matrix are all equal or unequal, which are described as follows $[5,6]$.

\section{A. Equal-eigenvalue case}

If all eigenvalues of the codeword difference matrix are 
equal, i.e. $\lambda_{1}=\ldots=\lambda_{K}=\lambda$, the PEP in (2) can be calculated as [5]:

$$
P_{e}(\mathbf{C} \rightarrow \hat{\mathbf{C}})=F\left(N_{r} K, u\right)
$$

where:

$$
\begin{gathered}
u=\sqrt{\frac{0.25 \lambda S N R}{1+0.25 \lambda S N R}} \\
F(N, u):=[0.5(1-u)]^{N} \sum_{k=0}^{N-1}\left[\left(\begin{array}{c}
N-1+k \\
k
\end{array}\right)(0.5(1+u))^{k}\right]
\end{gathered}
$$

with $N$ and $u$ being positive integer and real number, respectively.

\section{B. Unequal-eigenvalue case}

If the eigenvalues of $(\mathbf{C}-\hat{\mathbf{C}})(\mathbf{C}-\hat{\mathbf{C}})^{*}$ are not all equal, i.e. there are $K(K>1)$ distinct eigenvalues, then the PEP in (2) can be calculated as $[6]$ :

$$
P_{e}(\mathbf{C} \rightarrow \hat{\mathbf{C}})=\sum_{r=1}^{K} \sum_{s=1}^{N_{r}} A_{r, s} F\left(s, u_{r}\right)
$$

where:

$$
u_{r}=\sqrt{\frac{0.25 \lambda_{r} S N R}{1+0.25 \lambda_{r} S N R}}
$$

and $A_{r, s}$ is the factor of Partial Fraction Expansion (PFE) given by:

$$
A_{r, s}=\left.\frac{(-1)^{N_{r}-s}}{\left(\lambda_{r}\right)^{M-s}\left(N_{r}-s\right) !}\left\{\frac{d^{N_{r}-s}}{d(x)^{N_{r}-s}}\left[\prod_{k=1, k \neq r}^{K} \frac{1}{\left(1-x \lambda_{k}\right)^{N_{r}}}\right]\right\}\right|_{x=\frac{1}{\lambda_{r}}}
$$

Once the PEP of (2) is obtained using (3) or (6), the EUB on BER can be computed as:

$$
E U B=\frac{1}{L} \sum_{\mathbf{C}} \sum_{\hat{\mathbf{C}} \hat{\mathbf{C}} \neq \mathbf{C}} \frac{e(\mathbf{C}, \hat{\mathbf{C}})}{B} P_{e}(\mathbf{C} \rightarrow \hat{\mathbf{C}})
$$

where $L$ is the size of the codebook and $e(\mathbf{C}, \hat{\mathbf{C}})$ is the number of bit errors due to the error event $(\mathbf{C} \rightarrow \hat{\mathbf{C}})$ and $B$ is the number of bits per codeword.

\section{DIFFICULTIES IN USING THE EUB EXPRESSION}

Theoretically, the EUB on BER of (9) derived using the exact PEP expressions (3) and (6) is the tightest union bound and would be very useful for BER calculations and constructions of $\mathrm{S}-\mathrm{T}$ codes. However, we show here that it is not easy to accurately calculate the EUB on BER using (9). In calculating the EUB on BER of S-T codes, since the eigenvalues of the codeword difference matrix $(\mathbf{C}-\hat{\mathbf{C}})(\mathbf{C}-\hat{\mathbf{C}})^{*}$ are normally not all equal, equation (6) is more often used than (3). Moreover, at each SNR, it is required to calculate the exact PEP for $L(L-1)$ times in order to obtain the final EUB on BER, thus the accuracy in using (6) to calculate all these exact PEPs will surely affect the accuracy of the final EUB on BER. The expression in (6) mainly consists of two functions, $A_{r, s}$ and $F\left(s, u_{r}\right)$, given by (8) and (5), respectively, and so the tolerances on calculating these two functions affect the accuracy of the final EUB on BER.

\section{Difficulty to calculate $A_{r, s}$ for nearly equal eigenvalues}

It can be seen from (8) that $A_{r, s}$ has a derivative function of $N_{r}-s^{\text {th }}$-order. It can easily be proved that, after taking $N_{r}-s$ derivatives, a factor of $\frac{1}{\left(\lambda_{r}-\lambda_{k}\right)^{2 N_{r}-s}}$ will occur in the expression. When the value of $\lambda_{r}$ is very close to that of $\lambda_{k}$, the denominator of this factor will be small and hence the magnitude of the factor will be extremely huge. If $A_{r, s}$ is calculated numerically using computer, the computer program should be able to handle numbers with very large ranges; otherwise, the calculated PEP value would be inaccurate. Since the exact PEP expression of (6) is a summation of the products of $A_{r, s}$ and $F\left(s, u_{r}\right)$, where $F\left(s, u_{r}\right)$ is always positive and $A_{r, s}$ could be positive or negative, inaccurate calculations of $A_{r, s}$ could lead to negative PEP values which are meaningless in probability. From (9), the EUB on BER is obtained by averaging all possible PEPs, so negative PEPs could result in negative BER values. To illustrate this, we have written a $\mathrm{C}$ program to calculate the EUB on BER of a $4 \times 4$ MIMO system transmitting 8 QPSK symbols in a code-block length of 2 using (9). Figure 1 show the results for a range of SNRs when two different computer numbering formats were used in the $\mathrm{C}$ program. With the use of the float(32bits) type in the $\mathrm{C}$ program, Fig. 1a shows that more than half of the calculated values are negative which is meaningless in probability. Figure $1 \mathrm{~b}$ shows that the results are better when the computer numbering format is changed to the double (64bits) type, a higher precision format, but the problem could not be completely solved because there are still negative BER values.

Here we propose a trick to reduce the number of negative PEPs as follows. If the eigenvalues are close to each others, i.e., for $\lambda_{1}>\lambda_{2}>\ldots>\lambda_{k}$, and the following condition is satisfied:

$$
\left|\lambda_{1}-\lambda_{k}\right|<\mathcal{E}
$$

where $\mathcal{E}$ is a small value, then we simply assume that the eigenvalues are all equal and use (3) instead of (6) for PEP calculations. Since (3) always produces positive PEP values, the 
chance of having a negative BER value is reduced. Of course, more reduction can be obtained by increasing $\mathcal{E}$, but this will be at the expense of drop in accuracy. Results in the next section shows that the method can reduce the number of negative PEPs, but cannot eliminate all of them.

\section{Difficulty to calculate $F\left(s, u_{r}\right)$ at high SNR}

The value of $A_{r, s}$, as given by (8), is independent of the SNR. However, (5) indicates that $F\left(s, u_{r}\right)$ is a function of SNR through the function $u_{r}$ in (7). The function $u_{r}$ has a value between 0 and 1 and becomes very close to 1 at high SNRs. Equation (5) also indicates that $F\left(s, u_{r}\right)$ has a factor of $\left[0.5\left(1-u_{r}\right)\right]^{s}$ which becomes extremely small when $u_{r}$ is close to 1. To study the BERs of S-T codes, we are normally interested in the high SNR region where the BER is very small, thus the value of $F\left(s, u_{r}\right)$ needs a high precision computer to represent it accurately. To investigate the effects of $F\left(s, u_{r}\right)$ on the PEP calculation, we have written $\mathrm{C}$ programs to study the percentage of negative PEPs forming the corresponding BER values in Fig. 1. Results are shown in Fig. 2 which indicates that the percentages of negative PEPs are much higher at high SNRs. When the SNR is increased from $9 \mathrm{~dB}$ to $20 \mathrm{~dB}$, the percentage of negative PEPs is increased by 10 times. The percentage of negative PEPs is much reduced by using a higher precision computer numbering format, double type.

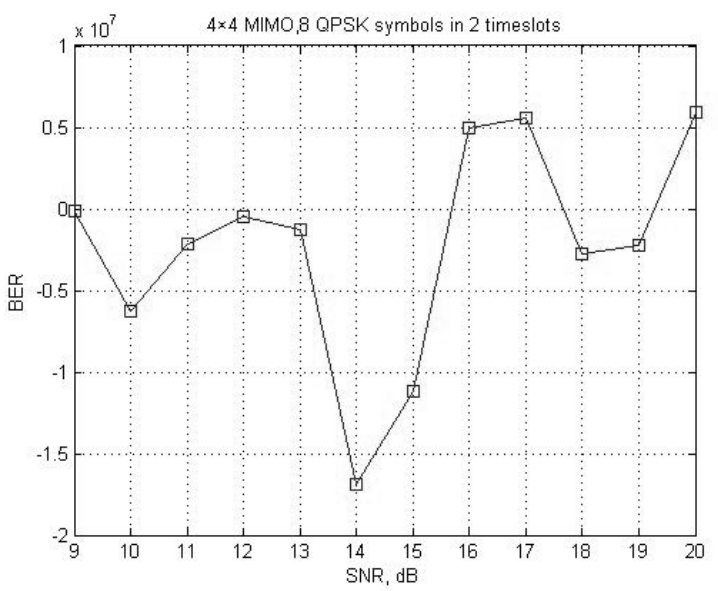

(a)

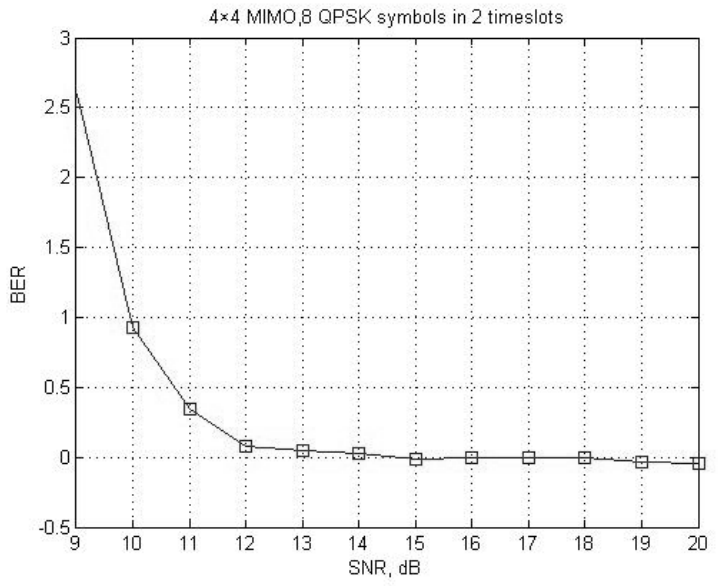

(b)

Fig.1. Numerically calculated EUB on BER using a) float and b) double types in $\mathrm{C}$ program

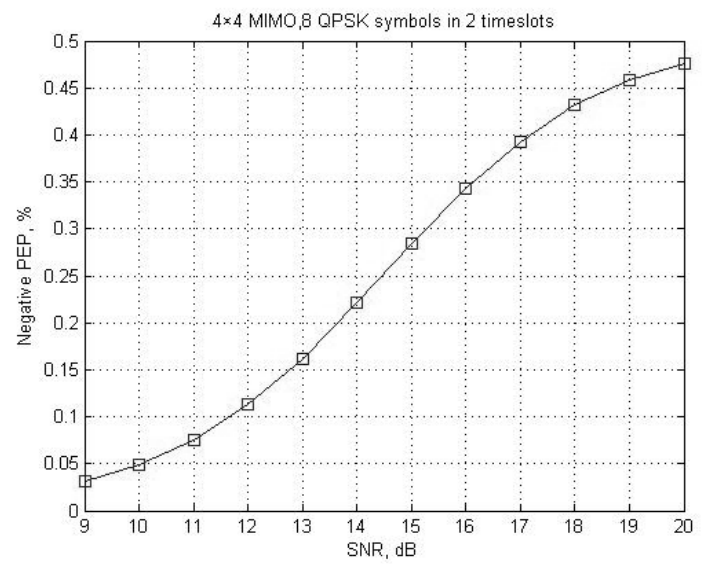

(a)

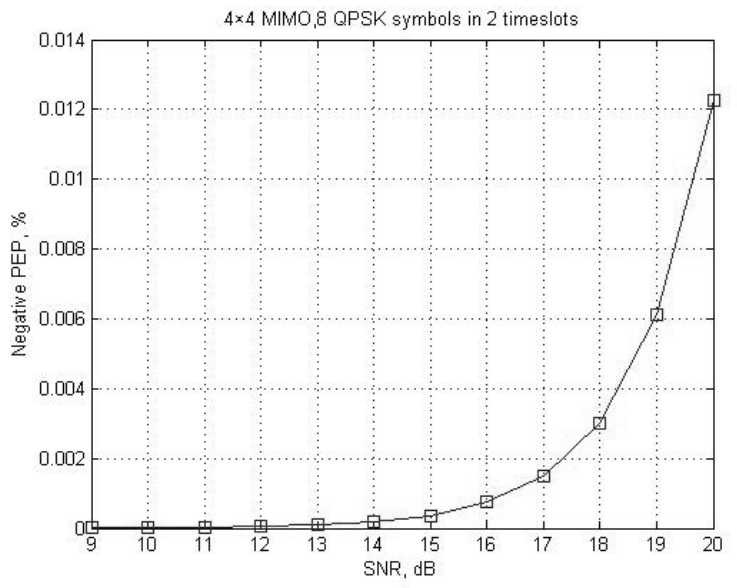

(b)

Fig. 2. Percentage of negative PEPs using a) the float and b) the double type in $\mathrm{C}$ program 


\section{NEW UNION BOUND ON PEP}

Using the HÖlder inequality in integral form [7], a new upper bound on PEP can be derived as:

$$
P_{e}(\mathbf{C} \rightarrow \hat{\mathbf{C}}) \leq\left[\prod_{r=1}^{K} F\left(N_{r} K, u_{r}\right)\right]^{\frac{1}{K}}
$$

where $u_{r}$ and $F\left(M K, u_{r}\right)$ are defined in (7) and (5), respectively. Based on the PEP of (11), we propose a new union bound (NUB) on BER:

$$
N U B=\frac{1}{L} \sum_{\mathbf{C}} \sum_{\hat{\mathbf{C}} \hat{\mathbf{C}} \neq \mathbf{C}} r(\mathbf{C}, \hat{\mathbf{C}})\left[\prod_{r=1}^{K} F\left(N_{r} K, u_{r}\right)\right]^{\frac{1}{K}}
$$

Different C programs have been written to apply (9) and (12) to calculate the union bounds on BER. The computer numbering format used in the $\mathrm{C}$ programs was double type. Figure 3 shows the BER results on the same MIMO system using the NUB, the EUB with the trick (described previously) and $\mathcal{E}=0.01$ and Monte Carlo simulation. For comparison purpose, the absolute values of the BERs using the EUB without the trick are also shown in the same figure. Monte Carlo simulation has been used to obtain the actual BER performance of the system and the result, as shown in Fig. 3, is used as a reference for measuring the performances of the union bounds studied. It can be seen in Fig. 3 that, with the use of our proposed trick, the EUB on BER looks normal up to the SNR of $16 \mathrm{~dB}$. However, it becomes abnormal when SNR $>16 \mathrm{~dB}$, so our proposed trick cannot solve the problem completely. At $\mathrm{SNR} \leq 16 \mathrm{~dB}$, our proposed NUB and the EUB using the trick perform about the same. At SNR $>16 \mathrm{~dB}$, our proposed NUB on BER is very close to the simulation result.

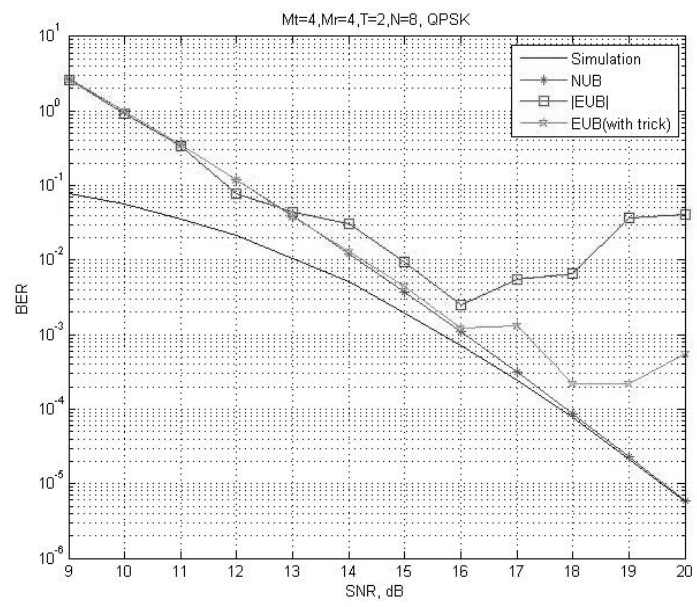

Fig. 3. Performance comparison

\section{CONCLUSIONS}

We have explained and presented the results to show that the exact closed-form PEP and the corresponding EUB formulae are not suitable for numerical calculations of BER. When the eigenvalues of the codeword difference matrix are very close to each others or the SNR is high, the calculation results using the EUB on BER are inaccurate and sometimes can be negative. A trick has been suggested to reduce the effects of the problem, but it still cannot be resolved completely. A new upper bound of PEP derived using the HÖlder inequality has been proposed to replace the exact closed-from PEP. Results have shown that the NUB based on the proposed upper bound of PEP is very tight to the EUB, but can be reliably computed using numerical calculation without having the same problem of the EUB.

\section{REFERENCES}

[1]. V. Tarokh, N. Seshadri, and A. Calderbank, "Space-time codes for high data rate wireless communication: Performance criterion and code construction," IEEE Trans. Inform. Theory, Mar.1998.

[2] M.K Byun and B.G. Lee, "New bounds of pairwise error probability for space-time codes in Rayleigh fading channels", 2002 IEEE WCNC, March 2002 Page(s):89 - 93 vol.1

[3] M.P Fitz, J. Grimm and S. Siwamogsatham, "A new view of performance analysis techniques in correlated Rayleigh fading", WCNC'99, IEEE, Sept 1999

[4] R.K Mallik and Q.T.Zhang, "A tight upper bound on the PEP of a Space-Time Coded system", IEEE trans on wireless communications, Sept 2007

[5] John Proakis, "Digital Communications, $4^{\text {th }}$ edition", New York: McGraw-Hill, 2001

[6] H.F Lu, Y.K Wang; P.V Kumar, K.M Chugg, "Remarks on space-time codes including a new lower bound and an improved code", IEEE Trans. Inform. Theory, Oct. 2003

[7] G.H Hardy, J.E Littlewood, G. Polya, "Inequalities", Cambridge, New York, 1934 